\title{
Metamaterials Fabricated by Fibre Drawing
}

Fleming, S.; Stefani, A.; Hayashi, J.; Kuhlmey, B.

Published in:

Proceedings of 23rd Opto-Electronics and Communications Conference

Link to article, DOI:

10.1109/OECC.2018.8730125

Publication date:

2018

Document Version

Peer reviewed version

Link back to DTU Orbit

Citation (APA):

Fleming, S., Stefani, A., Hayashi, J., \& Kuhlmey, B. (2018). Metamaterials Fabricated by Fibre Drawing. In Proceedings of 23rd Opto-Electronics and Communications Conference [8730125] IEEE. 23rd Opto-Electronics and Communications Conference, OECC 2018 https://doi.org/10.1109/OECC.2018.8730125

\section{General rights}

Copyright and moral rights for the publications made accessible in the public portal are retained by the authors and/or other copyright owners and it is a condition of accessing publications that users recognise and abide by the legal requirements associated with these rights.

- Users may download and print one copy of any publication from the public portal for the purpose of private study or research.

- You may not further distribute the material or use it for any profit-making activity or commercial gain

- You may freely distribute the URL identifying the publication in the public portal

If you believe that this document breaches copyright please contact us providing details, and we will remove access to the work immediately and investigate your claim. 


\title{
Metamaterials Fabricated by Fibre Drawing
}

\author{
S. Fleming*, A. Stefani*,**, J. Hayashi* and B. Kuhlmey*,*** \\ * Institute of Photonics and Optical Science (IPOS), School of Physics, University of Sydney, NSW, Australia \\ ** DTU Fotonik, Department of Photonics Engineering, Technical University of Denmark, DK-2800 Kgs. Lyngby, Denmark \\ *** Centre for Ultrahigh bandwidth Devices for Optical Systems (CUDOS), Physics, University of Sydney, Australia
}

\begin{abstract}
The fibre drawing technique is utilized for fabricating metamaterials. The ability to engineer both the permittivity and permeability is demonstrated. A hyperlens with $\lambda / 176$ sub-diffraction focusing is realized. Tunability of $50 \%$ is also demonstrated.
\end{abstract}

\section{INTRODUCTION}

Metamaterials can exhibit properties not found in natural materials, from which devices with extraordinary functions can be made. Invisibility cloaks [1] and subdiffraction imaging lenses are example devices. The extraordinary properties arise from the metamaterial microstructure which is a composite of precise subwavelength features, typically of metal and dielectric. Fabrication of bulk quantities of volume metamaterials is a challenge due to the combination of dissimilar materials, the scale, and the uniformity required throughout a macroscopic volume.

We have applied the fibre drawing technique to solve this fabrication challenge. This is a novel application of a well-established industrial microfabrication technique.

\section{Metamaterial FAbrication AND CharaCterisation}

\section{A. Engineered Permittivity}

The simplest form of metamaterial is a "wire array" [2] comprised of an array of fine wires within a dielectric. The permittivity depends very largely on the size and spacing of the wires, so is readily engineered.

Conceptually, our fabrication approach fills the air holes of a microstructured fibre preform with metal. Combining the Taylor wire process [3] with microstructured polymer optical fibre [4] fabrication, we use a polymer preform and fill the holes with indium, which is chosen because it is molten at the polymer drawing temperature.

We fabricated metamaterial fibres for use at terahertz ( $\mathrm{THz}$ ) frequencies by drawing this preform to fibre. Three different diameters were drawn, to demonstrate the scale

This work was supported by the Marie Sklodowska-Curie grant of the European Union's Horizon 2020 research and innovation programme (708860). The work was supported in part by Australian Research Council (ARC) Discovery Projects DP140104116 and DP170103537. This work was performed in part at the Optofab node of the Australian National Fabrication Facility (ANFF), using NCIRS and NSW State Government funding. dependence of permittivity. The fibre was cut into sections a few $\mathrm{cm}$ long and stacked together to make a planar sample. Figure 1 shows the transmission of these samples characterized using $\mathrm{THz}$ time domain spectroscopy (THz TDS). The boundary between transmission and reflection gives the plasma frequency, which determines the permittivity. As expected from theory, the measured plasma frequency changed with the geometry [5], demonstrating that fibre drawing can indeed be used to fabricate this class of metamaterials.

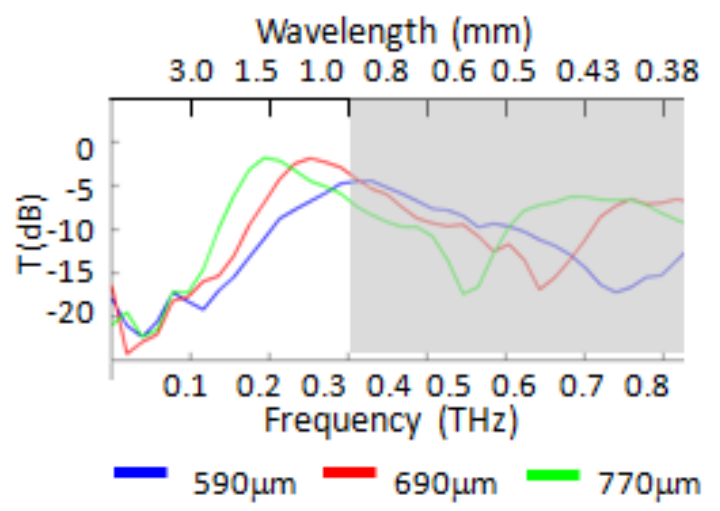

Fig. 1. Transmission versus frequency for metamaterial drawn to three different sizes, demonstrating shift of plasma frequency with scale [5].

These results were performed at $\mathrm{THz}$ frequencies largely because of the material we chose and the dimensions are easier to fabricate. We subsequently extended this range into the mid-IR [6].

\section{B. Engineered Permeability}

To engineer the permeability requires different shaped metallic inclusions, in this case slotted cylinders. We fabricated another type of metamaterial fibre by a similar process using slotted cylinders for single and double "split ring resonator" (SRR) structures [7].

These SRR metamaterials demonstrated strong magnetic resonances at frequencies just sub-THz, and permeabilities substantially different from unity, including negative values [8].

\section{Metamaterial HyperLenS}

Moving from demonstrating the ability to create materials with a wide range of permittivities and permeabilities we chose to fabricate a hyperlens to demonstrate a metamaterial device with important practical functionality. 
The wire array structure has an additional property: depending on the polarization of incident radiation it can behave as a dielectric or a metal. With this extreme anisotropy, radiation with polarization transverse to the wires can propagate without diffraction. With a tapered structure sub-diffraction focusing can be achieved.

We made two hyperlenses and concatenated them, as shown in Figure 2. The first hyperlens comprised 473 hexagonally spaced indium wires embedded in a PMMA/Zeonex fibre. The wire diameter through the tapered region reduces from $\sim 160$ to $\sim 20 \mu \mathrm{m}$, while their separation decreases from 400 to $50 \mu \mathrm{m}$. The second hyperlens comprised 462 hexagonally spaced tin wires embedded in a soda-lime glass fibre. The wire diameter of this tapered region reduced from 10 to $2.5 \mu \mathrm{m}$, while the separation decreased from 20 to $5 \mu \mathrm{m}$. The expected magnification factor of this hybrid hyperlens is $32 \mathrm{x}$ [9].

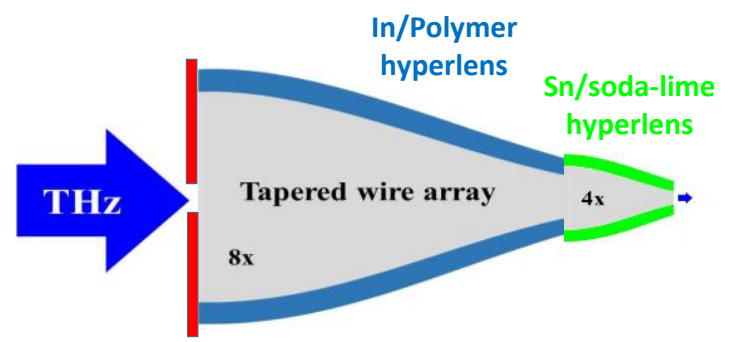

Fig. 2. Schematic of combined polymer and glass metamaterial hyperlenses used in the focusing experiment [9].

The focusing capability was characterized in the $\mathrm{THz}$ TDS, illuminating a $1.5 \mathrm{~mm}$ pinhole at the large end and scanning the near field at the small end. Measurements at three different frequencies, 0.034, 0.058 and $0.072 \mathrm{THz}$, showed the pinhole focused to $\sim 50, \sim 60$ and $\sim 70 \mu \mathrm{m}$ respectively, corresponding to sub-diffraction focusing of $\lambda / 176, \lambda / 87$ and $\lambda / 60$ respectively (see Fig. 3 for $\lambda / 176$ ).

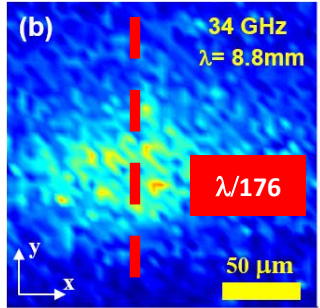

Fig. 3. Focused image of the pinhole after the concatenated hyperlenses, measured in near field at $0.034 \mathrm{THz}(\lambda \sim 8.8 \mathrm{~mm})$ [9]

\section{Tunable Metamaterials}

The properties of metamaterials depend very largely on their geometry, rather than materials, so tunability requires the ability to dynamically modify that geometry. For most fabrication approaches geometry is locked in when the device is made. Our approach creates an additional opportunity to make tunable devices. The dielectrics considered so far, such as PMMA and sodalime, have relatively high Young's moduli, in the GPa range, which does not permit much stretching or compression. We explored the use of polyurethane, with a Young's modulus in the MPa range [10]. As shown in
Fig. 4, the polyurethane based metamaterial can be easily compressed (using tweezers) and returns to its original shape when released.

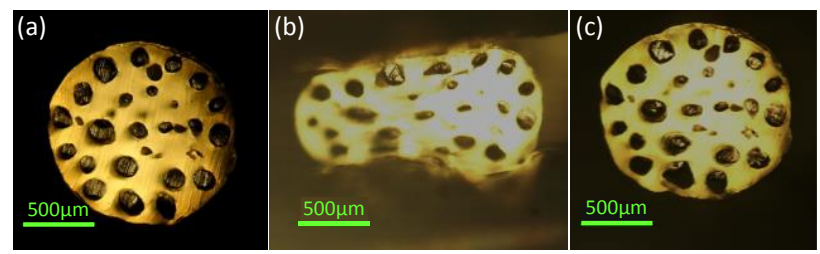

Fig. 4. Changing the geometry of the metamaterial using tweezers. (a) Original, (b) compressed, (c) released after compression [10].

When measured using THz TDS the plasma frequency shifted by around $0.2 \mathrm{THz}$ from $0.395 \mathrm{THz}$. This $\sim 50 \%$ tuning of the plasma frequency was consistent with a modelled 20-30\% compression of the structure. Importantly it was reversible and repeatable, demonstrating a viable approach to tuning metamaterials.

\section{Conclusions}

We have demonstrated that fibre drawing can be used to fabricate metamaterials with engineered permittivity and permeability for frequencies from mid-IR to $\mathrm{THz}$. We have applied this technique to realise extreme subdiffraction focusing up to $\lambda / 176$ with metamaterial hyperlenses, and demonstrated tunable metamaterials.

\section{ACKNOWLEDGMENT}

The authors acknowledge the fibre fabrication skills of R. Lwin and the contributions of many students.

\section{REFERENCES}

[1] D. Schurig et al., "Metamaterial electromagnetic cloak at microwave frequencies," Science, vol. 314, pp.977-980, 2006.

[2] J.B. Pendry et al., "Extremely low frequency plasmons in metallic mesostructures," Phys Rev Lett, vol. 76, pp.47734776, 1996.

[3] G.F. Taylor, "A Method of Drawing Metallic Filaments and a Discussion of their Properties and Uses," Physical Review, vol. 23, pp.655-660, 1924.

[4] M.A. van Eijkelenborg et al., "Microstructured polymer optical fibre," Optics Express, vol. 9, pp.319-327, 2001.

[5] A. Tuniz et al., "Drawn metamaterials with plasmonic response at terahertz frequencies," Applied Physics Letters, vol. 96, pp.191101, 2010.

[6] O.T. Naman et al., "Indefinite Media Based on Wire Array Metamaterials for the THz and Mid-IR," Advanced Optical Materials, 2013, 1(12) pp.971-977.

[7] N. Singh et al., "Fiber-drawn double split ring resonators in the terahertz range," Optical Materials Express, vol. 2 pp.1254-1259, 2012.

[8] A. Wang et al., "Fiber metamaterials with negative magnetic permeability in the terahertz," Optical Materials Express, vol. 1, pp.115-120, 2011

[9] J.G. Hayashi et al, "Extreme sub-diffraction focusing of THz radiation with magnifying metamaterial hyperlenses", Proc. of Australia New Zealand Conference on Optics and Photonics (ANZCOP), Queenstown (New Zealand) Dec. 2017, Paper 37

[10] S. Fleming et al, "Tunable Metamaterials Fabricated by Fiber Drawing", JOSA B, vol. 34, pp.D81-D85, 2017. 